\title{
COMUNICACIÓN
}

\section{Loxosceles laeta, identificación y una mirada bajo microscopía de barrido}

\author{
DEMITRI PARRA*; MARISA TORRES**; JOSE MORILLAS *** y PABLO ESPINOZA ****
}

\section{Loxosceles laeta, A LOOKING OVER SCANNING MICROSCOPE}

Loxosceles laeta is a spider of importance in Chile and produce hight morbilethality in humans. The majority of the patients with a spider bite go to primary attention and to the emergency room. It is very important to know the morphology of the spider in order to be prompt identify and make a correct management of the patients. We showed its morphology in a photography of scanning electronic microscopy that detach its most fundamental aspects, like its six eyes located in a triangular disposition.

Key words: Spiders, Loxosceles laeta, Loxoscelism, Araneida.

\section{INTRODUCCIÓN}

Las arañas del género Loxosceles pertenecen al orden Araneida, al suborden Labidogntha (se caracteriza porque sus quelíceros, tienen ubicación horizontal y al morder se entrecruzan como una pinza al cerrar) y a la Familia Sicariidae. Las arañas del género Loxosceles tienen una distribución mundial, siendo la especie $L$. laeta la de mayor distribución en Sudamérica, única en su género en Chile ${ }^{1}$ y sin duda la más tóxica y peligrosa ${ }^{2}$; la Loxosceles reclusa se encuentra en Norteamérica ${ }^{3}$ y la Loxosceles refescens en países Mediterráneos y en el Medio Oriente ${ }^{4}$. Esto no excluye que se hayan descrito mordeduras por otras especies como Loxosceles gaucho en Argentina y Loxosceles intermedia en Brasil ${ }^{5}$.

La mordedura de L. laeta constituye una patología relevante en nuestro medio por la magnitud de los casos y la alta morbimortalidad. En atención primaria y servicios de urgencia es una causa frecuente de consulta ${ }^{6-8}$, aunque el número exacto de mordeduras no se conoce, las estimaciones por registros parciales evidencian su importancia.

En la unidad Parasitología de la Universidad de Chile en 1955 se reportaron 230 casos de loxoscelismo ${ }^{9}$ y entre 1985 y 1995 hubo 43 muertes por mordedura de araña (L. laeta

\footnotetext{
* Interno de Medicina de la Pontificia Universidad Católica de Chile

** Médico Parasitólogo Pontificia Universidad Católica de Chile.

*** Licenciado en Ciencias Biológicas, Laboratorio de Microscopía electrónica de la Fac. de Ciencias Biológicas de la Pontificia Universidad Católica de Chile. QEPD año 2000.

****Encargado Laboratorio de Entomología de la Facultad de Ingeniería Forestal y Agronomía de la Pontificia Universidad Católica de Chile.
} 
Latrodectus mactan) con un rango entre 2 y 8 muertes anuales ${ }^{10}$.

Es importante familiarizarse con la morfología de esta arácnido pues el diagnóstico precoz de su mordedura permite intervenir terapéuticamente para prevenir complicaciones. Este artículo desea mostrar los aspectos morfológicos más relevantes de L. laeta con el objeto de ayudar al clínico a realizar un diagnóstico eficaz y oportuno, valorando la potencial gravedad del fenómeno.

\section{MATERIAL Y MÉTODOS}

Se capturaron arañas L. laeta en un recinto académico (detrás de cuadros y muebles) siendo seleccionado un ejemplar macho. Para conservar la integridad de las arañas, éstas se capturan vivas en forma diurna desde su hábitat natural. Se observó la araña, y se le colocó encima un frasco de vidrio transparente invertido, bajo el cuál se deslizó papel corriente con el objeto de cerrar su salida y evitar el escape.

El ejemplar vivo se revisó con lupa a través del frasco y posteriormente se fijó en formalina para observarlo bajo microscopía electrónica. Se fijó en formalina al $4 \%$ por un periodo de 6 horas, realizándose luego un lavado prolongado con agua corriente por 2 horas. Se procedió a realizar una deshidratación en alcohol con concentraciones crecientes (50, 70, 96 y $100 \%$ respectivamente). Posteriormente se practicó deshidratación con acetona en concentraciones crecientes $(50,70,90$ y $100 \%$ respectivamente). Luego el espécimen se llevó a un aparato de secado a punto crítico (SPC), realizándose sombreado con oro y platino en un "sputtering". La preparación se montó en un portamuestra y se observó en microscopio electrónico de barrido Geol $25 \mathrm{~S}$. con $30 \mathrm{KV}$.

\section{DESCRIPCIONES}

La L. laeta en su estado adulto mide entre 8 y $15 \mathrm{~mm}$ de longitud y sus patas entre 8 y $30 \mathrm{~mm}$. Morfológicamente tiene dos segmentos claramente visualizados; cefalotórax y abdomen. Es de color café pardusco (Figura 1), y esta cubierto por abundantes pelos largos y erectos y de pelos acostados y ramificados desde la base hasta la mitad de su longitud. En los tarsos de las patas hay pelos que cumplen funciones táctiles.

El cefalotórax tiene un aspecto piriforme (cuerpo de violín) con tres pares de ojos simples distribuidos en forma de triángulo (dos pares laterales y uno anterior), lo que caracteriza al género ${ }^{13}$. Sus quelíceros o dientes inoculadores están adheridos desde la base hasta la parte media de su cara interna. El margen interno, quitinizado, se prolonga hacia delante, formando una punta aguda sobre la cual apoya el ápice de la uña articulada. Tiene una forma arqueada y desarrolla un movimiento de bascula. (Figura 2).

Posee además un aparato estridulatorio, del tipo quelícero palpo, que aparece en los primeros estados de maduración, teniendo una función reproductiva. En la copula, una vez que el macho y la hembra han establecido contacto continuo, la hembra entra en estado de catalepsia, levanta el cefalotórax y sus primeros pares de patas son llevados a los costados gracias a que el macho con el segundo par se apoya en la cara inferior del primer par de la hembra y el tercer par del macho bajo el segundo par de la hembra; el macho extiende los palpos (aparato estridulatorio) rectamente hacia adelante, introduciéndolos simultáneamente en el aparato femenino, del modo que parece ser característico de las haploginas (arañas que no poseen órganos femeninos exteriores). La introducción dura unos pocos segundos y puede repetirse unas tres o cuatro veces. (Figura 2).

Sus extremidades están formadas por un fémur, tibia, metatarso y tarso, que carecen de formaciones especiales, y salvo su longitud, son similares entre machos y hembras. El tarso es pequeño, tan largo como ancho y de una forma aproximadamente cúbica.

Debido a que son arañas haploginas no existen órganos femeninos exteriores que indiquen que las hembras han alcanzado un estado adulto. Sin embargo, cierto oscurecimiento en el cefalotórax y una mayor nitidez en el pliegue epigástrico, permite advertir al ojo experimentado cuando una hembra adquiere su madurez sexual. Los caracteres que indican la edad madura de un macho son la transformación del palpo en aparato copulador, y el aumento de 


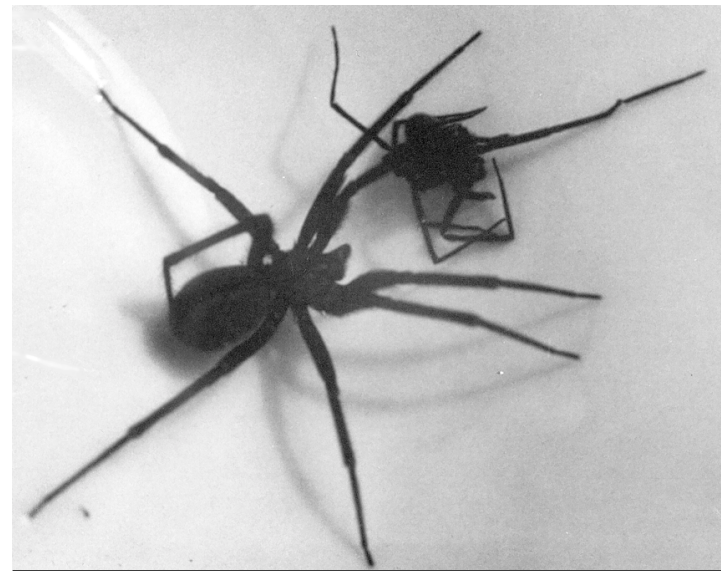

Figura 1. Ejemplar adulto de Loxoceles laeta. Sin aumento.

longitud de sus patas..$^{14}$

Esta araña tiene dimorfismo sexual y desarrolla una metamorfosis incompleta es decir después de una reproducción sexuada de un macho y una hembra, la hembra coloca los huevos en ootecas que contienen en promedio 88,37 huevos (mínimo 29 y máximo 138) blanquecinos amarillentos y de diámetro entre 1.166 a $1.249 \mathrm{~mm}$. Estos, dan origen a pequeñas arañas que después de tres estados (9 a 12 mudas) y en un tiempo de 315 días para hembras y 406 días para machos, llegan al estado adulto. En el laboratorio se ha observado el número promedio en días de vida: 696 para muchos que se han apareado, 1.155 para machos vírgenes; 1.536 para hembras fecundadas y 1.894 para hembras vírgenes. ${ }^{15,16}$

La araña se alimenta de moscas, polillas y pequeños artrópodos, los cuales caza en la oscuridad o atrapa en su tela. Su toxina se almacena en un aparato venenoso ubicado en el cefalotórax, compuesto por glándulas continuas, revestidas por un epitelio secretor y rodeadas por células musculares. Cuando es sorprendida por la luz del día o la luz artificial busca refugio en el primer lugar que encuentra que puede ser ropa colgada en la pared, camas, toallas, etc. Generalmente la mordedura a una persona es una situación accidental y se produce cuando la araña es presionada contra la piel de la víctima. ${ }^{17}$

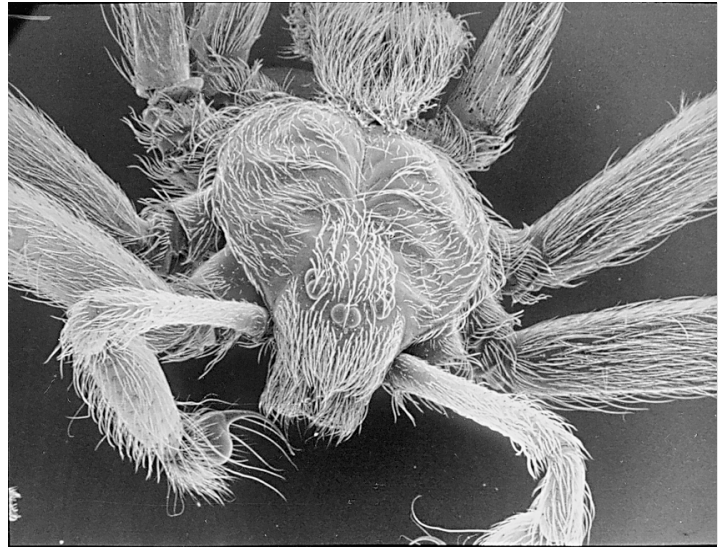

Figura 2. Aumento: barra $=100$ micrones. Visión anterior de cefalotórax que permite observar visión anterior de aparato estridulatorio y los 3 pares de ojos.

\section{DISCUSIÓN}

Para el médico en atención primaria y servicio de urgencia en Chile, ante un cuadro de picadura de artrópodo (insecto o arácnido), es útil observar al artrópodo (muerto) bajo lupa simple. Si se determina que posee cuatro pares de patas (arácnido) debe observar el color del espécimen y el tamaño: si es café y no posee mancha en el abdomen se debe sospechar $L$. laeta, en tanto si la araña es de un tono más oscuro con una mancha rojiza o amarillenta en el abdomen se debe sospechar que se trata de L. mactans, la cuál provoca un cuadro clínico muy específico en un contexto epidemiológico particular (adulto, zona rural).

Luego debe observarse el cefalotórax, para lo que se recomienda pasar un algodón húmedo por la zona para desplazar los pelos e ir a visualizar la distribución de los ojos. La $L$. laeta que sería la de mayor importancia tiene la distribución de ojos ya señalada.

Se debe educar a la población que cuando sea picado por algún artrópodo, intente llevarlo (vivo o muerto) o restos de él, pues ayudará al diagnóstico oportuno.

\section{RESUMEN}

Laxosceles laeta es una araña de importancia en Chile por su alta morbiletalidad en hu- 
manos. La gran mayoría de los pacientes con mordedura por esta araña consultan en servicios de atención primaria y de urgencia. Es relevante conocer su morfología para identificarla en forma precoz y tener una conducta médica adecuada a seguir. Se ilustra su morfología con fotos de microscopía de barrido destacando sus aspectos más fundamentales entre ellos sus seis ojos dispuestos en forma triangular.

\section{REFERENCIAS}

1.- SCHENONE H. Loxoscelismo cutáneo de predomino edematoso. Bol Chil Parasitol 1998; 53: 7883.

2.- FUTRELL J. Loxoscelism. Am J Med Science 1992; 304: 261-7.

3.- HARRISON et al. Principles of Internal Medicine. Mc Graw-Hill Companies Inc. 14 th Edition. 1998; Vol. 2: 2551.

4.- BORKAN J, GROSS E, LUBINH Y et al. An outbreak of venomous spider bites in a cutrus grove. Am J Trop Med Hyg 1995; 52: 228-30.

5.- TAMBOURGI D V, MAGNOLI F C, VAN DEN BERG $C$ et al. Sphingomyelinases in the venom of the spider Loxosceles intermedia are responsible for both dermonecrosis and complement-dependent hemolisis. Biochem Biophys Res Commun 1998; 25: 366-73.

6.- HEREDIA V, ARBAIZA E, VENEGAS $\mathrm{J}$ et al. Aporte al estudio de las acciones proteolítica, procoagulante y caracterización electroforética de las proteínas de dos extractos tóxicos de veneno de Loxosceles laeta. Bol Chil Parasitol 1989; 44: 8-16.
7.- SALAS M, ZAVALETA A. Toxicidad aguda del veneno glandular de Loxosceles sp. en ratones albinos. Bol Chil Parasitol 1986; 41: 43-51.

8.- MARLON U, ZENNIN M, KOWLASKI L et al. A clinical and epidemiological study of Loxosceles spider envenoming in Santa Catarina, Brazil. Trans R Soc Trop Med Hyg 1998; 92: 546-8.

9.- SCHENONE H. Diagnóstico hechos a 1.384 pacientes que consultaron por probable mordedura de araña o picadura de insectos. Bol Chil Parasitol 1996; 51: 20-7.

10.- MINISTERIO DE SALUD DE CHILE, SERVICIO DE SALUD M. CENTRAL, SUBDIRECCION MEDICA, UNIDAD DE EPIDEMIOLOGIA. Informe de loxoscelismo. Boletín informativo a los servicios de salud. Int $\mathrm{N}^{\circ} 067 \mathrm{~F}$ : 09.04.98.

11.- SCHENONE H, RUBIO S, VILLARROEL $F$ et al. Epidemiología y curso clínico del loxoscelismo. Estudio de 133 casos causados por la mordedura de la araña de los rincones (Loxosceles laeta). Bol Chil Parasitol 1975; 30: 6-17.

12.- GALIANO M. Ciclo Biológico y desarrollo de Loxosceles laeta. Acta Zoológica Lilloana XXIII, 1967.

13.- PARRA D, TORRES M. Loxoscelismo: una patología vigente. Pediatría al Día 2000; 16: 178-83.

14.- ATIAS A. Parasitología clínica. Editorial Mediterráneo, Santiago de Chile. Tercera Edición. 1991; 556-61.

15.- SCHENONE H, LETONJA T. Notas sobre la biología y distribución geográfica de las arañas del género Loxosceles. Bol Chil Parasitol 1975; 30: 27-9.

16.- GALIANO M, HALL M. Datos adicionales sobre el ciclo vital de Loxosceles laeta. PHYSIS Sección C., Buenos Aires. 1973; 32: 277-88.

17.- SCHENONE H, SAAVEDRA T, ROJAS A et al. Loxoscelismo en Chile. Estudios epidemiológicos, clínicos y experimentales. Rev Inst Med Trop Sao Paulo 1989; 31: 403-15. 